\title{
Optimizing the complexities of unforeseen risk in healthcare with innovation and technology: a proposed framework
}

\author{
Asfandyar Khan ${ }^{1 \wedge}$, Aimen Farooq ${ }^{2}$, Sarfraz Ahmad $^{3}$, Jane M. Fraser ${ }^{4}$ \\ ${ }^{1}$ AdventHealth Daytona Beach, Daytona Beach, FL, USA; ${ }^{2}$ AdventHealth, Orlando, FL, USA; ${ }^{3}$ AdventHealth Cancer Institute/AHMG, FSU and \\ UCF Colleges of Medicine, Orlando, FL, USA; ${ }^{4}$ Department of Engineering, Colorado State University-Pueblo, Pueblo, CO, USA \\ Correspondence to: Asfandyar Khan, MSc. AdventHealth Daytona Beach, Daytona Beach, FL 32117, USA. Email: Asfandyar.khan@adventhealth.com.
}

Received: 12 January 2021; Accepted: 24 April 2021; Published: 25 March 2022.

doi: $10.21037 /$ jhmhp-21-3

View this article at: http://dx.doi.org/10.21037/jhmhp-21-3

\section{Introduction/background}

The United States (U.S.) healthcare system is a subject of debate. Fifteen years ago, the U.S. healthcare system was declared almost broken, and it has not improved since (1). U.S. citizens spend much more money on healthcare than any other country in the world and critics point out inefficiencies. It is also argued that Americans have the best healthcare system in the world with access to freely available medical technologies and state-of-the-art facilities (2). However, weaknesses become more critical when a pandemic hit, and now every country in the world seems to be in the same boat.

The U.S. National Academies, Advisor to the Nation on Science, Engineering and Medicine, reported on July 20, 2005 that "The U.S. healthcare industry has neglected engineering strategies and technologies that have revolutionized quality, productivity, and performance in many other industries, says a new report from the National Academies' National Academy of Engineering and Institute of Medicine. This 'collective inattention' has contributed to serious consequences in healthcare nearly 100,000 preventable deaths per year, outdated procedures, about a half-trillion U.S. dollars wasted annually through inefficiency, costs rising at roughly three times the rate of inflation, and 43 million people uninsured. Healthcare professionals and engineers should work more closely together to address these challenges, said the committee that wrote the report (3)."

Quality care and efficiency are the main requirements of a healthcare system. Usually, more staff or equipment is added by hospital decision makers to improve efficiency because insufficient resources are thought to be the main cause of the problem. However, the added resources increase the cost. The challenge for the stakeholders is to understand that there is need to develop systems in healthcare sector to optimize the unforeseen scenarios, future demand and supply of healthcare workers, facilities, and research capabilities. Creating an ideal healthcare system is a challenge due to many factors, most importantly financial, but there are always recommended strategies where the daily healthcare operations can be optimized. The destabilization of certain departments within the healthcare system encountering unforeseen scenarios takes away essential resources and creates a vacuum of workforce in other healthcare departments. Quality healthcare has become a worldwide goal. Societies around the world have become increasingly intent on actualizing the value proposition in healthcare, and the science of healthcare quality is advancing rapidly (4).

In this manuscript, the normal operation of a medium size healthcare facility (i.e., taking care of around 1,400 patient per week on normal operation days pre COVID-19) was studied and compared with the ongoing pandemic condition in the world. The case study shows how reactive strategies from the unforeseen situation affected the normal operations, created a vacuum for the workforce, and choked the financial situation of the healthcare system. Preliminary analyses indicate that a balance within the different health departments was lost which resulted in inefficiencies,

$\wedge$ ORCID: 0000-0002-9649-9269. 
shortage of workforce in certain medical areas, higher cost and that it will require a long time to recover. Stakeholders must understand the concepts and learn the skills involved in designing their organization to exploit their inherent strengths (5).

This paper also recommends a proposed framework for how the U.S healthcare can develop better systems and proactive strategies in the healthcare sector when the unforeseen risk scenarios develop. Strategies include balancing healthcare workers, research scholars demand and supply for different communities, developing facilities in due course of time and optimizing the lean supply for medical equipment in a pandemic situation to reduce overall cost. Physicians should take time to understand the key features of law such as MACRA and the opportunities it presents to shape the future of payment and medical practice (6). It has been observed that when a situation develops, some of the financial budget is wasted in medical supplies which further results in holding costs and there is little left for the onground healthcare workers. Adequate medical supplies are always essential when dealing with a pandemic condition if modular manufacturing facilities are locally available. The paper specifically addresses two aspects: (I) what will be the "new normal" for the U.S healthcare industry, and (II) how can the healthcare system keep running normal daily operations with scenarios of unforeseen conditions. We discuss that (i) creating basic proactive strategies will strengthen the health industry, (ii) developing and maintaining the systems requires time, and (iii) the timeline for each system can be developed and optimized based on the individual community adaption level.

\section{Historical perspectives}

Delay in healthcare delivery can be very expensive and is often subject to heavy cost as well as dangerous to the patient's health and quality-of-life. The cost and delay problem can be addressed by developing better approaches. The U.S healthcare system is dependent on financial payments. Although health facilities are available to all, when people without healthcare insurance receive healthcare and cannot pay back, the cost is shared by other people who are paying into the healthcare system or by the government stakeholders. If the allocated funds are used to keep the current system functioning, it is a financial challenge to generate funds for future medical research, unforeseen risks, scenarios, and systems.

Healthcare spending in the United States in the year
2008 rose to $\$ 2.4$ trillion (16\% of Gross Domestic Product, GDP), with a projection of $\$ 3.1$ trillion in the year 2012, and $\$ 4.3$ trillion by the year 2016 (7). By the year 2017, the healthcare expenditures reached $20 \%$ of the GDP. In comparison, the Organization for Economic Cooperation and Development (OECD) reports that healthcare spending accounted for $10.95 \%$ of the GDP in Switzerland, $10.7 \%$ in Germany, 9.7\% in Canada, and 9.5\% in France (8). Nearly 46 million Americans are uninsured, but the U.S spends more on healthcare than any other industrialized nations that provide universal health coverage to all their citizens (9).

The first phase attempted to understand the rate of arrival of patients and the types of patients arriving on daily basis in a hospital depending on the rural or metropolitan area (pre-COVID-19 time). In a rural area with a population of approximately 50,000 if there is a hospital in the community, the average rate of arrival of patients is 24 patients every day depending on the type of care available. In a metropolitan community with a population of approximately 150,000 , the average arrival rate can be 286 patients every day depending on the type of care available. The average rate of arrival can increase or decrease due to stochastic circumstances happening each day in the communities. Most of these patients arriving the hospital are walk-in, means they arrived by their own means of transportation to be seen by physicians. Depending on the location of the hospital, there is $12 \%$ chance of patients arriving by ambulance and that percentage can increase or decrease with the type of community and transportation mode available to patients.

The second phase is understanding the hospital operation (pre-COVID-19), different scenarios and emergency severity index (ESI) levels. In order to better understand the severity levels, we divide the arriving patient into five different severity levels with "severity index 1 " as the most critical requiring maximum hospital resources (for example: one or more physician, nurses, bed, MRI, blood, intubation, rapid surgery, or medical procedures) and "severity index 5 " the least critical requiring minimum hospital resources. Patients rated as the ESI levels 1 and 2 are considered "high-risk" and patients rated between the ESI levels 3 to 5 are considered "low-risk". Patient recovery and condition can fluctuate within the severity levels depending on the health conditions. Table 1 shows the risk category correspondence to the ESI levels. The purpose of triage in the emergency department (ED) is to prioritize incoming patients and to identify those who cannot wait to be seen depending upon the initial ESI (10). 
Once a patient arrives in the hospital and is seen by the physician, each patient is assigned an ESI level depending on the current medical condition and laboratory test results. Historical data shows that most patients coming to the hospital lie between the ESI levels of 3 and 4. Including patients who require ambulatory or inpatient surgeries, and patients who require an adequate amount of physician's observation time before they can be discharged. For most patients of the ESI levels 3 and 4, the length-of-stay (LOS) at a healthcare facility is a maximum of 48 to 60 hours. Figure 1 depicts the historical occurrence of the five ESI levels and the percentage of patients in different ESI on an average day in a hospital. Figure 1 can also be used for any hospital in a region from small to medium or medium to large depending upon their daily rate of the arrival of patients. Approximately $80 \%$ of the patients in a hospital

Table 1 Emergency severity index (ESI) Levels

\begin{tabular}{lc}
\hline ESI levels & Category \\
\hline 1 & Very high risk \\
2 & High risk \\
3 & Moderate risk \\
4 & Low risk \\
5 & Minimal risk \\
\hline
\end{tabular}

are the ones who require emergency, internal, or family medicine care. Approximately 10\% of the patients require surgeries or high-level sub-specialty care, and the rest of the $10 \%$ are the lowest ESI patients who can go home on the same day after appropriate treatment and/or a management plan is discussed.

Apart from these severity levels, the hospital and healthcare system also run thousands of laboratory tests (for example: patients vitals, screening, pre-admission tests, oncology and histology tests, etc.), outpatient services for regular routine appointments, scheduled procedures in surgery as well as non-essential and emergency procedures. Hospitals and healthcare systems nationwide rely on the revenue that comes from the elective or non-essential procedures, such as stents, angioplasty, hysterectomy, outpatient services, procedures that are necessary but may not be emergencies or high-risk.

Furthermore, understanding the operational condition (pre-COVID-19 time) of the U.S. healthcare system requires analyzing the demand and supply of new physicians in the community, which is affecting the doctor-to-patient ratio. While a 'doctor shortage' has been reported, from the year 2010 to 2019, the actively licensed U.S. physician-topopulation ratio increased from 277 to 295 physicians per 100,000 people (11). The U.S. faces a looming shortage of physicians and nurses, constraints of facilities, low funding

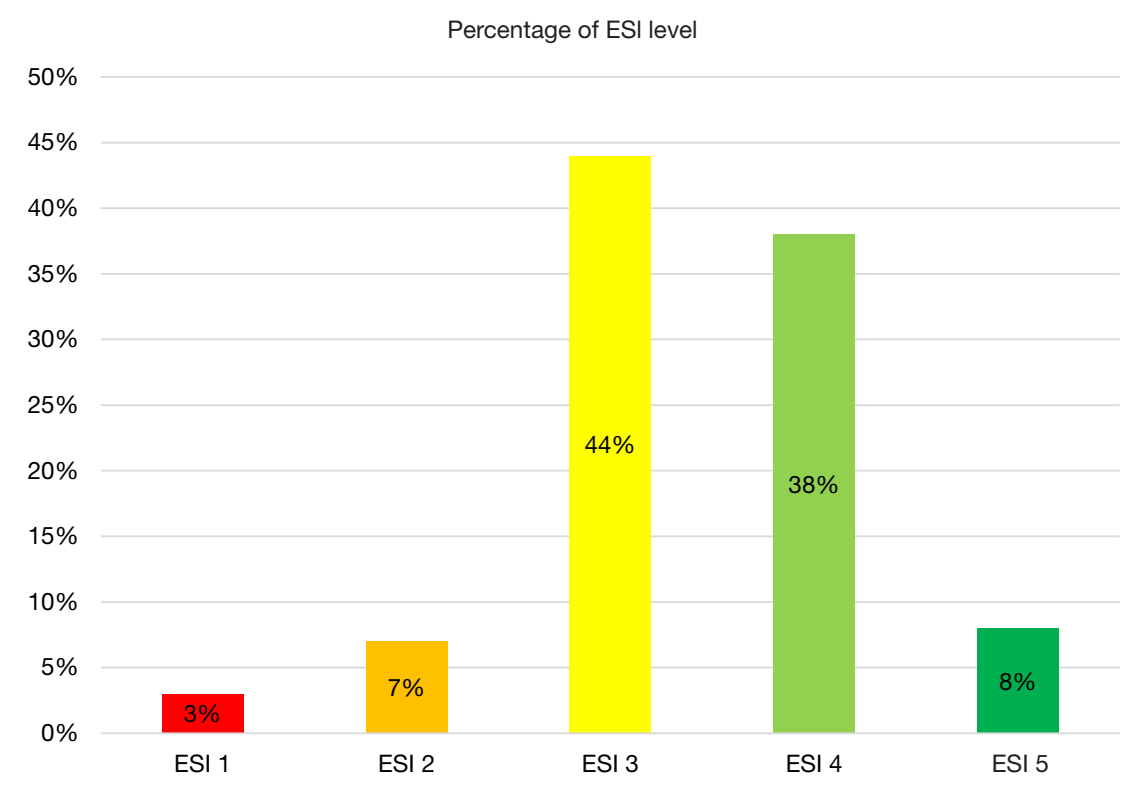

Figure 1 The percentage of patients in accordance with their ESI level on daily basis in a hospital/healthcare facility. ESI, emergency severity index. 
for research, an increasing rate of patient arrival, and higher LOS problems. The hospital and healthcare sectors run their medical supplies on lean management principles with a running inventory of a maximum of two to three weeks supply. Healthcare sector material management departments predict medical supplies on a weekly demandand-supply model, which is a linear model without any stochastic conditions monitored. Most of the basic medical supplies are acquired globally due to the cost and the criteria for quality. Local industries have neglected the need to produce the medical supplies due to raw material and high labor cost issues.

Now that we have described the above two phases of the healthcare sector, let us consider an unforeseen risk of ESI level 6 or a pandemic (the recent ongoing COVID-19 situation is an example) adding to the healthcare sector. Although there were early recommendations from different key players, the lack of acceptance and understanding of the global and local research by the stakeholders led to the use of reactive action policies rather than a proactive strategy. With almost no preparation for the unforeseen risk, the stakeholders' reaction was to shut down the communities and non-essential services in the healthcare sector. This reactive and sudden policy was put into action as some communities started to show a surge in patients' arrival rate. The initial shutting down helped the healthcare workers understand the new severity level which they were dealing with at an unpredictable rate. The cancellation of most "non-essential" procedures and outpatient services were meant to support any patient surge due to the nonavailability of facilities and medical staff required. The focus was to create a special ward for patients for COVID-19 having limited and unexperienced manpower who are still in the process of learning about the virus but have the courage to serve. The stakeholders' reaction methodology disrupted the current healthcare system and its lean methods by just focusing on the COVID 19 control. The justification was simply "no one was prepared". This methodology affected many of surgical as well as outpatient employees in the form of pay cuts and imposed furloughs. Some medical departments were left with doctors without patients. As the healthcare industry is run by earning through different other procedural departments, whose patients come from the ESI levels 1, 2, 3, 4 and 5 as explained in Figure 1, this unforeseen scenario also highlighted the shortage of certain physicians in the affected medical field.

The condition also caused researchers, analytical scholars, and healthcare management to develop reactive models. The unforeseen scenario requires real time data, which most of the time is stochastic and requires extensive industrial engineering methods to accept and/or reject the hypothesis. Risk models were developed within the communities and healthcare industry. A surge of patients in some communities created panic, which resulted in developing similar models in some cases without gathering and using any data on the results of actual testing. Stakeholders at different levels lack the understanding on how to interpret the confidence interval of any risk model. In some cases, no one questioned the source, validity, and verification of the data.

Millions of dollars of personal protective equipment and medical supplies were ordered globally by healthcare management without understanding and challenging the confidence intervals of predictive models. The holding cost in the form of excessive medical inventory has also created future financial challenges. Each model created possibilities and situations for future operational and new normal routine, but the operations kept changing every day or every week. Stakeholders with little confidence predicted new timelines and the risks of low manpower in respective areas and increased patient flow remains. The American Hospital Association estimates a total four-month financial impact of \$202.6 billion in losses for America's hospitals and health systems, or an average of $\$ 50.7$ billion per month. The federal government moved quickly to provide relief; more help is needed (12).

Closing different procedural departments has already created a backlog and a financial crisis in healthcare sectors. The patient arrival rate in hospital emergency and healthcare facilities decreased during the initial closure whereas in some facilities the numbers were out of control. A shortage of staff willing to work under critical circumstances was highlighted. Re-opening the communities in different phases also created a huge flow of patients that were waiting for elective procedures and follow up appointments; the question remains how to balance the "new normal" and prepare for the unforeseen future accordingly.

The United States, Center for Disease Control and Prevention (CDC) frequently studies and proposes responses for different disease models. A pandemic like COVID-19 was foreseen and planned for, but stakeholders haven't been successful in using those plans. There was an initial failure of the U.S. health care system to properly activate plans in place to deal with a pandemic like this. One of the most important factors that led to the 


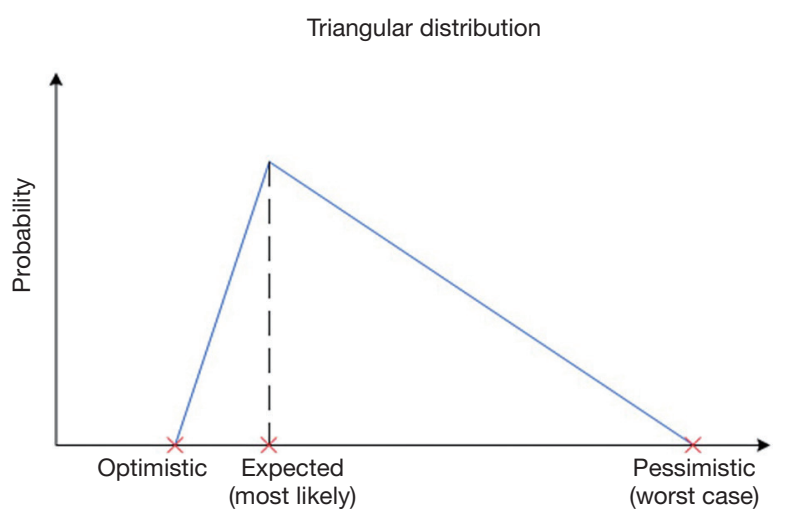

Figure 2 Triangular distribution for unseen scenarios in healthcare system with no historical data.

failure of activating response plan was not reviewing or updating policies since 2016 (13). Risk based analysis for an organization operating under ISO 9001 has been given a far more prominent role in the 2015 standard than it received in the past (14). In order to respond better to the next pandemic, the stakeholders must identify the risks and opportunities in the US health system. Research funding is needed for planning responses, and for verifying and validating acceptance level of responses using simulation models, and for integrating the response into the frontline leaders' actions by updating policies. Lastly the systems must be audited the systems to evaluate effectiveness.

\section{Proposed framework}

The healthcare system needs to be flexible, which means it should be able to handle a variety of requirements in different communities depending on the types of care available. It needs to be modular, operating at different efficiency levels and at different rates. It must be adaptable and able to respond to disruption. It must be kept functioning and operating at any flow and in any unforeseen conditions. With the healthcare system that we have, we do not wish the system to stop working. We are at a point where healthcare in the United States is getting more attention from law makers, government agencies, doctors, hospitals, pharmaceutical companies, and the population at large (15). Optimizing the resources available and balancing the healthcare sector operation in any new scenario will come with preparing an educated workforce solely focused on research, building remote facilities in due course of time, and preparing the workforce to support unforeseen conditions in healthcare sector. Medical supplies are important but can still be streamlined with predictive models, relying on the local industries, and creating proactive demand with safety supplies. Three main systems are recommended to be developed by the government stakeholders as well as private sectors to support the country's future healthcare demands.

\section{Surveillance in bealthcare}

With the above scenario in mind, imagine that everything goes back to normal or the "new normal", but the risk of developing new scenarios in healthcare sector remains and will keep increasing in the future. Adding any new severity level to the existing severity level conditions is always a possibility and an ongoing challenge. The percentage of patients in the new severity level can be anything from $1 \%$ to $99 \%$. When the data on time required for completing a task is not available and/or data gathered may not be appropriate to collect for the patient's and doctor's privacy, an alternative method can be used to estimate the probabilities but requires a higher confidence interval. Without having any historical data and prediction, researchers mostly use the triangular distribution where we express expert judgement in worst-case, optimistic, and most likely scenarios. Figure 2 shows how the expected trend can be adjusted between the optimistic and pessimistic realities.

Local and global healthcare surveillance is important in predicting any new scenario cases. Healthcare scenarios are stochastic; hence the forecast, hypothesis, and confidence interval change every second with the changing condition(s). The first step stakeholders need to understand and develop is to create a healthcare surveillance system locally and globally. Healthcare surveillance requires actual and factual studies. Satellites and electronic data can be helpful in generating the population models and the population transfer rates in and out of a region, but healthcare surveillance depends on the experimental dataset, extensive research on good and bad viruses, and medical research on how to control these viruses. Vaccine researchers and pharmaceutical scientists require information and live virus access in a controlled environment to generate the cure strategies. The timeline for these processes requires a great deal of engineering strategies and interventions. There are many contributing engineering disciplines like robotics, metallurgy, information technology, digital arts and science, 


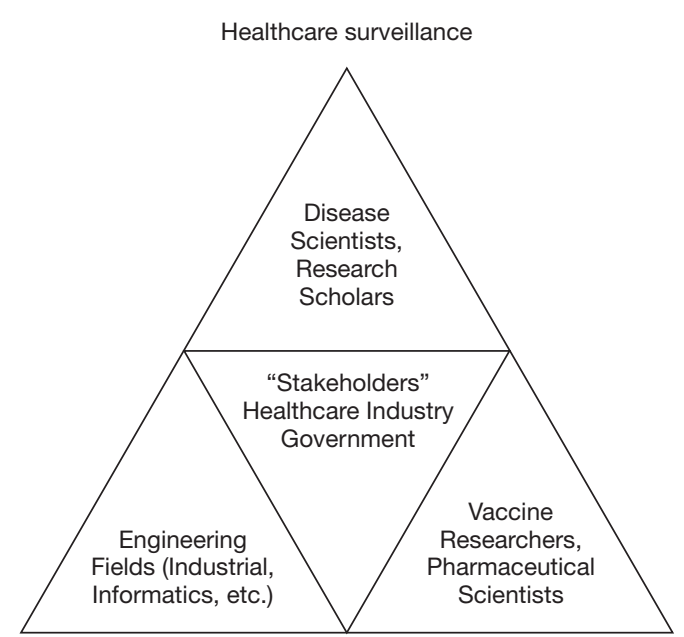

Figure 3 Healthcare surveillance system.

and the field of industrial and systems engineering. These engineering fields have a long history of improving the structure, processes, and outcomes of complex systems in many industries. Figure 3 depicts the key players in healthcare surveillance. Stakeholders' proactive decision depends on the following three key players:

(I) Disease scientists and research scholars.

(II) Vaccine researchers and pharmaceutical scientists.

(III) Engineering researchers and scientists.

The healthcare industry has generally neglected engineering strategies and technologies that have revolutionized quality, productivity, and performance improvements in many other industries (16). Funded programs need to be developed at school levels to train and encourage people interested in stochastic healthcare surveillance. These programs should share the surveillance data with healthcare professionals locally and globally to predict the percentage of any new severity level. Stakeholders must have the capability and understanding on how to challenge the data source validity and verification. In recent years, some attention has been driven to modeling, simulation, and optimization techniques capable of representing and improving discrete event systems. These techniques can support decision making helping to determine the best scenario on a combinatorial search space with stochastic variables (17). Stakeholders must also have education on creating a confidence level to accept or reject these models. This surveillance will lead to proactive strategies from stakeholders in developing the new structures, processes, complex systems to maintain the balance within the healthcare industry.

\section{Workforce in bealthcare}

Physicians (and nurses) are at the core of healthcare sector, as they make it possible for everyone to understanding the severity of a new scenario and they help us survive with better quality of care. The second aspect/recommendation is to create a healthcare workforce to balance the daily operation. Any new scenario will require healthcare workers. The rate and type of patient percentage will determine as to what kind of workforce is required to balance the operational needs. The government and healthcare stakeholders need to understand the percentage of patients in accordance with their severity level daily arriving in hospitals. Stakeholders also need to understand the shortage of certain types of physicians in medical fields verses the probability of patient arrival type and the unforeseen severity level. Thus, understanding the doctorto-patient ratio in each state as well as in each community level is critical. There is a need to create more programs to graduate more medical doctors as well as programs for nurses, phlebotomists, and healthcare laboratory researchers to support the unforeseen risk scenarios.

The recent/ongoing pandemic (COVID-19) has created a learning opportunity for the U.S. to streamline its healthcare industry. It should be a calculated risk of developing and investing in new programs all over the universities/institutions as well as communities where new medical graduates can learn, practice, and develop skills to serve the communities. The government and communities need to stand up for a cause and invest in such programs where they can develop a healthcare manpower so that when pandemic situations occur, the system is flexible, modular, adaptable, and operable at any unforeseen conditions/demands.

One of the key aspects here is perhaps developing graduate medicine residency programs all over the U.S. to overcome the supply and demand of physicians in the required fields. Every year, a reasonable number of physicians are retiring with a wealth of experience and they are looking for communities where they can live the rest of their life transferring the skills. However, the lack of teaching facilities is creating a gap not only in the rural but also in the metropolitan areas, which results in reactive decisions when a pandemic hit in order to balance the expected demands. Figure 4 depicts the dependence of the top to bottom workforce in healthcare system and quantity of each workforce required. Projections of future supply and demand for physicians suggest a shortfall of 46,100 to 


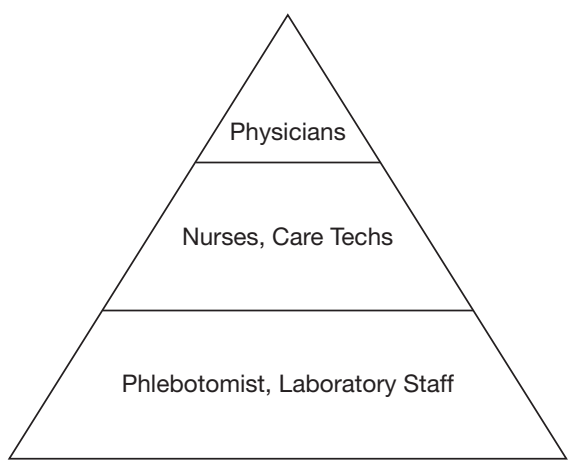

Figure 4 Workforce in healthcare system.

90,400 physicians, including a shortfall of 12,500 to 31,100 primary care physicians and a shortfall of 28,200 to 63,700 non-primary care physicians (18).

The second recommendation is to better develop programs for nurses, phlebotomists, and diagnostic laboratory staff. Physicians define and analyze problems, but they rely on the laboratory test results to control and cure the severity of the problems. There is a shortage of laboratory staff (for example; medical assistants, general laboratory technicians, microbiologists, and histologist technicians, etc.) who process and set the ground-limits so that physicians can read/interpret any new scenarios as recommended by the biomedical researchers and support staff. Creating a workforce who can support physicians in the reliability of laboratory processes is essential. Currently, the emerging shortage of Registered Nurses poses a real threat to the nation's health care system (19). With the current population growth rate and to support the desired doctor-to-patient ratio, the U.S. will soon require almost twice the number of nursing and laboratory staff as compared to the currently available (20).

\section{Modular facilities in bealthcare}

A pandemic situation (e.g., the recent/ongoing COVID-19 situation) creates a local and global closure, which means less, or in some cases, no transportation available. The third recommendation for the stakeholders is to create programs and alliances for the industrial companies within the communities to support the local medical research facilities and medical supplies. The alliances among the local industry will create industrial ability to survive the recession caused by any pandemic. Alliances among industries need to focus on their modular factory designs and the raw material required to support the healthcare industry needs. The goal of the plan should be to transform the U.S. healthcare sector from an underperforming conglomerate of independent entities into a high-performance "system" in which participating units recognize their interdependence and the implications and repercussions of their actions on the system as a whole (21).

Pandemic situations change the forecasting too often and too soon. Quarantined work forces and the paucity of manufacturing components are impacting the manufacturers across the globe. Local and global transportation delays affect lean procurement for the healthcare industry. Frustration among the stakeholders often results in unpleasant decisions, which can cause financial loss. The healthcare industry needs to develop relatively short distance and easy transportation plans for conditions like the COVID-19 pandemic. Modular industrial alliances are the need of the future to support the healthcare industry. Manufacturing needs to focus on the production of essential pharmaceuticals and medical equipment. Mechanical and garment factories need to broaden their horizon to create the possibilities to manufacture medical supplies and kits when in high demand. These recommendations are required to maintain the normal daily operation of the healthcare industry and for the future to balance out any pandemic condition. Figure 5 depicts how the stakeholders need to focus on balancing the facilities in healthcare system.

The local and global surveillance team needs to map out the best locations for developing these facilities depending on the present and future population growth and transportation rate. Location models are required based on minimizing the transportation cost or by single mean of the transfer rate within the local communities. One of the most important avenues is the test laboratories and testing sites. Physicians rely on the laboratory results; turn-aroundtime of the laboratory results have a huge impact on the treatment. Partnerships between rural hospitals and regional tertiary and academic health systems need to be advanced. Through these partnerships, rural hospitals can leverage the resources and capabilities for fulfilling their needs (22). The recent scenario of COVID-19 has highlighted the urgency of the experimental/clinical testing within the different population region as well their accuracy of the testing results. Surveillance is required to map out the community healthcare facilities, remote facilities, laboratories, testing, and research sites. Surveillance is also required to map out the location models to build the reusable healthcare 


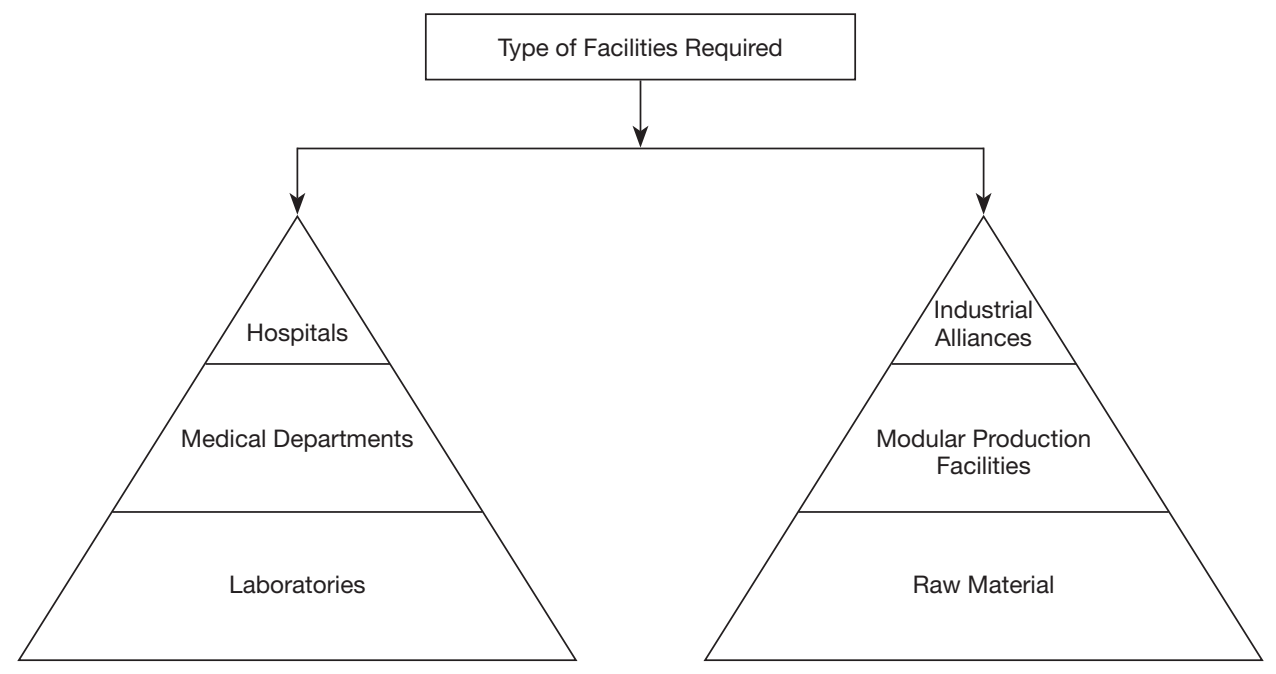

Figure 5 Balancing the facilities in healthcare system.

facilities within the due time to support the conditions like COVID-19 when required in certain regions. Information needs to be gathered and transferred to stakeholders, who must make the best decisions depending on the facts to support future growth and balance in the healthcare industry. Figure 6 depicts a proposed flow diagram for the balanced healthcare industry projection for the year 2020 and beyond.

\section{Conclusions}

The U.S. healthcare system may not be the best in the world, but it is better than many other countries. It is better at least to have a system and, as is said, there is no ideal system; there are systems and there is always room for improvement in systems or for developing new optimized future systems. Healthcare is filled with highly educated people working in an often-emotional environment, and respect is essential when developing a new business performance system (23). Stakeholders in the healthcare sectors need to develop systems and strategies as soon as possible to support the healthcare system beyond the year 2020. The timeline for each system can be trimmed accordingly but investments in healthcare systems are essential at this time. "Scientist and policymakers are operating on considerably different timescales. Normally, research programs take months and years, whereas policy decisions take weeks and months, sometimes days. This discrepancy makes it much more difficult to get scientifically generated knowledge into the policymaking process" (24).
Delaying investment will result in higher cost. The aim must be developing healthcare that's flexible, modular, adaptable, and operable at any unforeseen conditions.

\section{Summary}

Today, healthcare must be willing to take risks while advancing clinical transformation, starting from research to innovations, patient care technicians to physicians, management thinking lean to maximizing inventory flow, building a supply chain of healthcare workers to allowing engineering strategies, and building community facilities to establishing remote reusable facilities. In recent months, the ongoing pandemic (COVID-19) has changed everyone's perspective globally. The United States healthcare system is linked with financial needs. The stakeholders must understand the growth and possibility of the unforeseen new medical risks, including how to challenge the dataset used for decision making, increasing the required acceptance level for the verification and validation of growing medical risk models, because healthcare is a stochastic system, forecasting always changes and so does the final decision of investing the cost. The global fear brought on by the ongoing pandemic is playing a major role in the economic and social consequences. Experts recommend that physicians must be willing to take over the key roles and lead these strategies, but at the same time better integration of engineering fields can play a huge role in helping physicians to understand the strategies. It is very important to help them craft a solution that healthcare workers can 


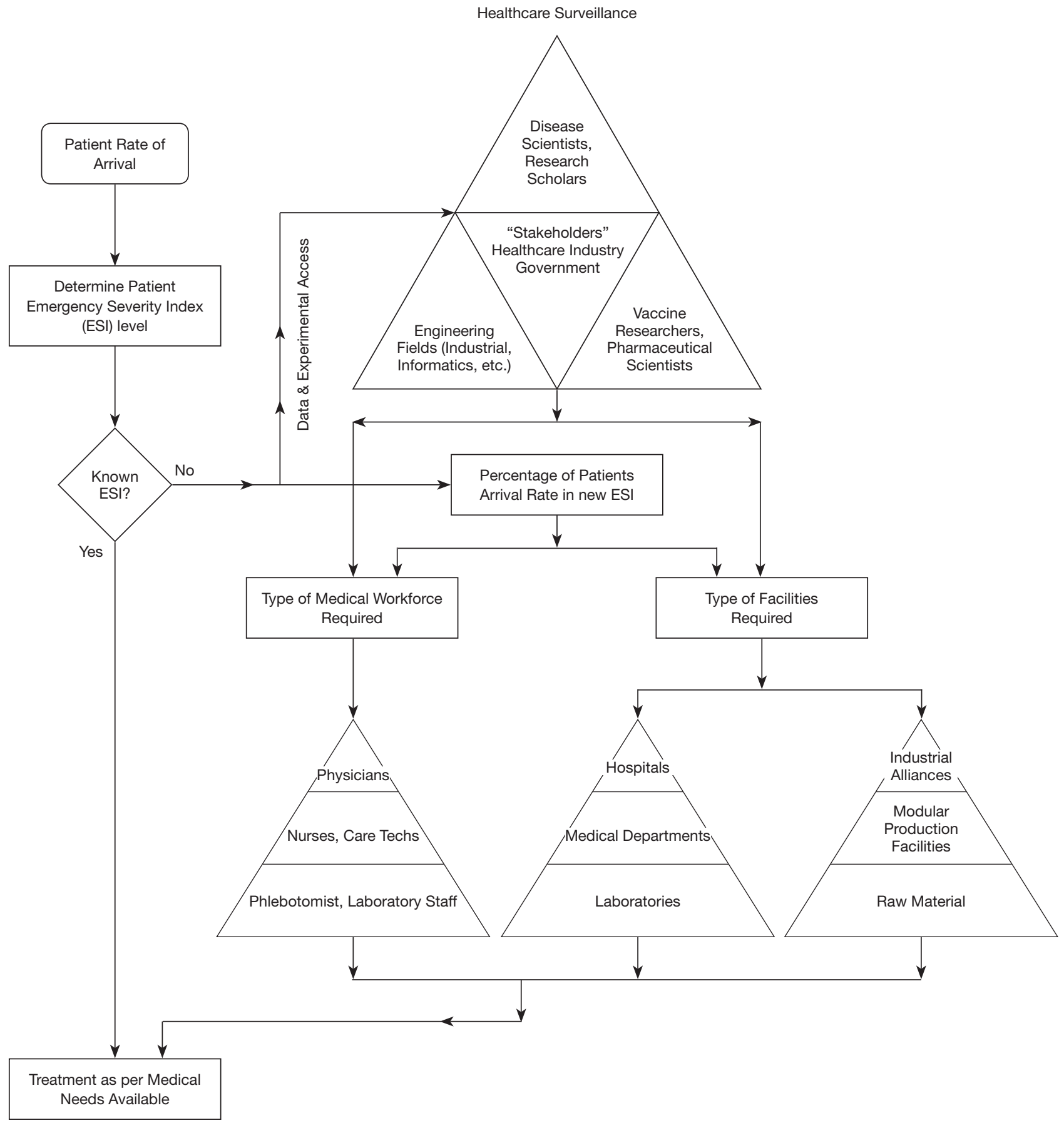

Figure 6 A proposed flow diagram for balanced healthcare industry. Projection 2020 and beyond. ESI, emergency severity index.

stick to. In this article, we propose three key frameworks (viz., health surveillance, workforce, and modular facilities) that would be helpful in creating a balance within the healthcare industry daily operation which is the paramount need of the "new normal" and sustainability.

\section{Acknowledgements}

The authors wish to thank the management of the healthcare organization and related laboratories for their help in this research. 
Funding: None.

\section{Footnote}

Provenance and Peer Review: This article was a standard submission to the journal. The article has undergone external peer review.

Peer Review File: Available at https://jhmhp.amegroups.com/ article/view/10.21037/jhmhp-21-3/prf

Conflicts of Interest: All authors have completed the ICMJE uniform disclosure form (available at https://jhmhp. amegroups.com/article/view/10.21037/jhmhp-21-3/coif). The authors have no conflicts of interest to declare.

Ethical Statement: The authors are accountable for all aspects of the work in ensuring that questions related to the accuracy or integrity of any part of the work are appropriately investigated and resolved.

Open Access Statement: This is an Open Access article distributed in accordance with the Creative Commons Attribution-NonCommercial-NoDerivs 4.0 International License (CC BY-NC-ND 4.0), which permits the noncommercial replication and distribution of the article with the strict proviso that no changes or edits are made and the original work is properly cited (including links to both the formal publication through the relevant DOI and the license). See: https://creativecommons.org/licenses/by-nc-nd/4.0/.

\section{References}

1. Garson A Jr. The US healthcare system 2010: problems, potential solutions. Circulation 2000;101:2015-6.

2. Chua K. Overview of the US health care system. Publication of the American Medical Student Association through the Jack Rutledge Fellowship. 2006.

3. The National Academies. Reported on July 20, 2005. Accessed April 10, 2020. Available online: http://nationalacademies.org

4. Lighter DE. Advanced Performance Improvement in Health Care: Principles and Methods, Jones \& Bartlett Learning, Massachusetts, MA, 2009:446.

5. Nadler DA, Tushman ML. Competing by Design: The Power of Organizational Architecture: Oxford University Press, New York, NY, 1997.

6. Clough JD, McClellan M. Implementing MACRA:
Implications for Physicians and for Physician Leadership. JAMA 2016;315:2397-8.

7. Keehan S, Sisko A, Truffer C, et al. National Health Expenditure Accounts Projections Team. Health spending projections through 2017: the baby-boom generation is coming to Medicare. Health Aff (Millwood) 2008;27:w145-55.

8. Pear R. U.S. Health care spending reaches all-time high: 15\% of GDP. The New York Times January 9, 2004:3.

9. California Health Care Foundation - Health Care That Works. Available online: https://www.chcf.org/ and https://www.chcf.org/publications/?fwp_topic=3559\&fwp_ paged $=3$

10. Gilboy N, Tanabe P, Travers D, et al. Emergency Severity Index (ESI): A triage tool for emergency department care, Version 4. Implementation Handbook 2012 Edition. Rockville, MD: Agency for Healthcare Research and Quality Publication No.12-0014, 2012:112.

11. Website: Doctor to Patient Ratio Wikipedia. Accessed April 17, 2020. Available online: https://en.wikipedia.org/ wiki/Physicians_in_the_United_States

12. American Hospital Association. Hospitals and Health Systems Face Unprecedented Financial Pressures Due to COVID-19. Accessed April 9, 2021. Available online: https://www.aha.org/guidesreports/2020-05-05-hospitalsand-health-systems-face-unprecedented-financialpressures-due

13. CDC page is dated 2016. Accessed September 30,2020. Available online: https://www.cdc.gov/flu/pandemicresources/planning-preparedness/index.html

14. ISO 9001, Risk Assessment Checklist. Accessed September 30, 2020. Available online: https://www.iso-9001-checklist. co.uk/how-to-address-risk-in-ISO-9001.htm

15. Chakravarthy SR. Stochastic Modelling in Healthcare Systems. In Proceedings of 1st International Conference on Simulation and Modeling Methodologies, Technologies and Applications: Simultech, 2011;1:109-15.

16. Valdez RS, Ramly E, Brennan PF. Industrial and systems engineering and health care: Critical areas of research. Final Report. (prepared by Professional and Scientific Associates under Contract No. 290-09-00027U.) AHRQ Publication No. 10-0079. Rockville, MD: Agency for Healthcare Research and Quality, May 2010.

17. Wilson TSJr, Montevechi JAB, Miranda RC, Campos AT. Discrete simulation-based optimization methods for industrial engineering problems: A systematic literature review. Comput Indust Enrg 2019;128:526-40.

18. Dall T, West T, Chakrabarti R et al. The Complexities 
of Physician Supply and Demand: Projections from 2013 to 2025 Final Report - Association of American Medical Colleges. 2015.

19. Caron VF. The Nursing Shortage in the United States: What Can be Done to Solve the Crisis? Seminar Research Paper Series. 2004; Paper 22. Available online: http:// digitalcommons.uri.edu/lrc_paper_series/22

20. Cox P, Willis WK, Coustasse A. The American Epidemic: The U.S. Nursing Shortage and Turnover Problem. Chicago, IL: Marshall University Digital Scholar Publication, Paper presented at BHAA 2014.

21. Proctor PR, Compton WD, Grossman JH, et al. editors. Building a better delivery system: A new engineering/ health care partnership. Publication of the Committee on Engineering and Health Care System, National Academy

doi: 10.21037/jhmhp-21-3

Cite this article as: Khan A, Farooq A, Ahmad S, Fraser JM. Optimizing the complexities of unforeseen risk in healthcare with innovation and technology: a proposed framework. J Hosp Manag Health Policy 2022;6:8. of Engineering, Institute of Medicine, 2005.

22. Mosley D, DeBehnke D. Rural hospital sustainability: New analysis shows worsening situation for rural hospitals and residents. Navigant Consulting, Inc., 2019; pp. 6. Accessed April 10, 2020. Available online: https://guidehouse.com/-/ media/www/site/insights/healthcare/2019/navigant-ruralhospital-analysis-22019.pdf

23. Barnas K Adams E. In: Beyond Heroes: A Lean Management System for Healthcare. Toussaint J, Womack J (Editors). ThedaCare Center for Healthcare Value, Appleton, WI, 2014.

24. Kwon D. Science and policy collide during the pandemic. The Scientist, September 1, 2020. Available online: https:// www.the-scientist.com/careers/science-and-policy-collideduring-the-pandemic-67882 